\title{
Observation of Pain-Sensitive Points along the Meridians in Patients with Gastric Ulcer or Gastritis
}

\author{
Hui Ben, ${ }^{1}$ Liang Li, ${ }^{1}$ Pei-Jing Rong, ${ }^{1}$ Zhi-Gao Jin, ${ }^{2}$ Jian-Liang Zhang, ${ }^{1}$ \\ Yan-Hua Li, ${ }^{1}$ and $\mathrm{Xia} \mathrm{Li}^{3}$ \\ ${ }^{1}$ Institute of Acupuncture and Moxibustion, China Academy of Chinese Medical Sciences, Beijing 100700, China \\ ${ }^{2}$ Beijing Coal General Hospital, Beijing 100028, China \\ ${ }^{3}$ Beijing University of Traditional Chinese Medicine, Beijing 100029, China
}

Correspondence should be addressed to Pei-Jing Rong, rongpj@mail.cintcm.ac.cn

Received 20 August 2012; Accepted 14 October 2012

Academic Editor: Wolfgang Schwarz

Copyright (๑) 2012 Hui Ben et al. This is an open access article distributed under the Creative Commons Attribution License, which permits unrestricted use, distribution, and reproduction in any medium, provided the original work is properly cited.

\begin{abstract}
This study aims to investigate the sensitization of human skin points along certain meridians related to visceral disease by using the pressure-pain threshold (PPT) as an indicator. We detected and compared the PPTs of people with and without gastric ulcer or gastritis on the related acupoints, abdomen area, and back area with von Frey detector and observed the similarities and differences under their respective physiological and pathological states. The results showed that (1) the PPTs of patients with gastric ulcer on related acupoints decreased significantly compared with the control group; (2) there was no significant difference in PPT between the chosen points of the measured meridian and the adjacent nonacupoints; (3) there was an apparent distribution of tender points on the relevant abdomen and back regions of patients with gastric ulcer or gastritis, but none was found on the control group; (4) the pain-sensitive points of gastric ulcer and gastritis patients were BURONG (ST19), LIANGMEN (ST21), and HUAROUMEN (ST24) of the stomach meridian on the abdominal region and PISHU (BL20), WEISHU (BL21), and WEICANG (BL50) on the back, among others The results suggest that the practical significance of acupoints may lie in its role as a relatively sensitive functional area. In a pathological state, the reflex points on the skin which are related to certain visceral organs become sensitive and functionally intensify.
\end{abstract}

\section{Introduction}

The pressure-pain threshold (PPT) of skin is the minimum force applied on the skin by external pressure which induces pain. It is one of the traditional measures to quantitatively evaluate pain that has been widely used in basic as well as clinic studies. In most cases, reporting of perceived pain by the subjects is usually influenced by factors like the expectancy, and pressure algometry however provides objective information of the local pain conditions.

According to the theory of meridians in traditional Chinese medicine, there are connections between the internal organs and their respective somatic meridians. Therefore, visceral lesions can lead to changes of pain sensation, which exhibited as tenderness point(s), or pigmentations and so forth in certain areas of the body surface. And similarly, the stimulation of acupoints can result in the regulation of function of the responding internal organs.
Previous studies have shown that the sensitive points on the body surface accompanied with some visceral disorders including the digestive diseases which are characterized with abdominal pain and tenderness as the common sign and symptom. In this study, by the help of von Frey detector, we measured the PPT of acupoints along meridians of subjects with gastric ulcer or gastritis to assess their sensitization under the pathological conditions.

\section{Materials and Methods}

\subsection{Detection of Pressure-Pain Threshold}

2.1.1. Detection Equipment. The 2390-type von Frey detector produced by the IITC Company of the United States was used to detect the PPTs of acupoints along the related meridians so as to compare the similarities and differences 


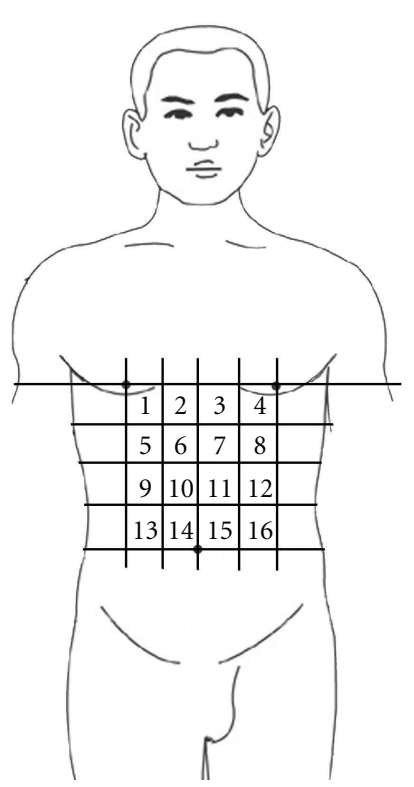

(a)

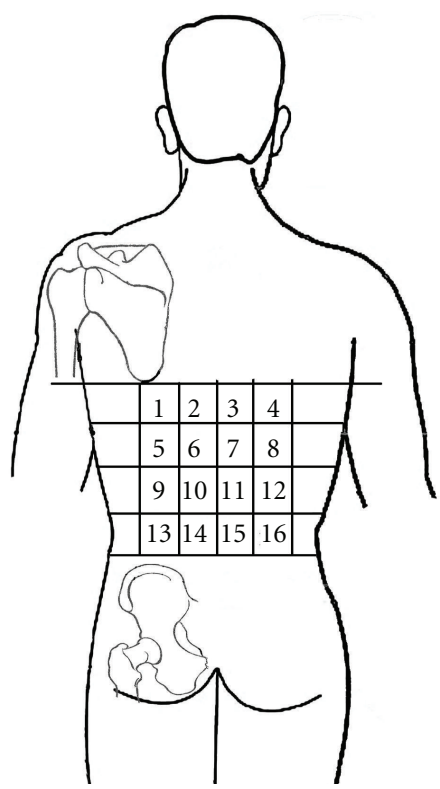

(b)

Figure 1: Abdominal and back testing zones. The abdominal and back regions of the subject were divided into 16 testing zones, respectively.

between the results acquired in normal and pathological states.

2.1.2. Detection of Acupoints. Tested acupoints (1) Stomach Meridian: ZUSANLI (ST36), SHANGJUXU (ST37), XIAJUXU (ST39); (2) Large Intestine Meridian: SHOUWULI (LI13), QUCHI (LI11), SHOUSANLI (LI10), HEGU (LI4); (3) back acupoints: WEISHU (BL21), DACHANGSHU (BL25); (4) points which are $1.0-1.5 \mathrm{~cm}$ adjacent to the above acupoints were selected as the control acupoints; (5) the abdominal and back acupoints.

2.1.3. Methods. The probe tip was kept moving vertically downward toward the skin at an even speed. When the subject felt the pain, the probe was removed immediately and the data on the detector were recorded simultaneously. Each point was tested three times at an interval of three minutes. The average of the data was taken as the threshold.

2.2. Partition of the Testing Zones on the Abdomen and Back. The abdominal and back regions of the subject were divided into 16 testing areas, respectively (Figure 1). In each testing area three points were tested, and each point had a certain distance to the other two points.

2.3. Identification of Tender Points on the Abdomen and Back. Tender points in the testing zones of the subject's abdomen and back were identified by finger-pressure method. Tender points were determined when the subject expressed obvious pain or a quasistringy pain while the thumb pressed vertically downward at an even speed.
2.4. Selection of Pain-Sensitive Points on the Abdomen and Back. Data are expressed as means of the values acquired from the three test points in each of the 16 testing zones mentioned above. The three points with the lowest pain threshold (i.e., pain-sensitive point) were selected and marked in Figure 5. The points marked for each group were cumulatively displayed in Figure 5 to demonstrate the different responses to pain of the relevant areas on the surface of body between healthy people and patients with gastric ulcer or gastritis.

\subsection{Grouping}

2.5.1. Control Group 1. Twenty healthy volunteers were selected (10 male and 10 female, aged 25-50). The PPTs were measured, respectively, on the stomach meridian, large intestine meridian, back meridian, and those adjacent to open acupoints.

2.5.2. Gastric Ulcer Group 1. Sixteen volunteers with gastric ulcer were selected ( 8 male and 8 female, aged 30-55). The PPTs were measured, respectively, on the stomach meridian, large intestine meridian, back meridian, and those adjacent to open acupoints.

2.5.3. Control Group 2. Twenty one healthy volunteers were selected (11 male and 10 female, aged 30-55). The PPTs were measured, respectively, in each testing zone on the abdomen and back.

2.5.4. Gastric Ulcer Group 2. Sixteen volunteers with gastric ulcer were selected ( 8 male and 8 female, aged 30-55). 


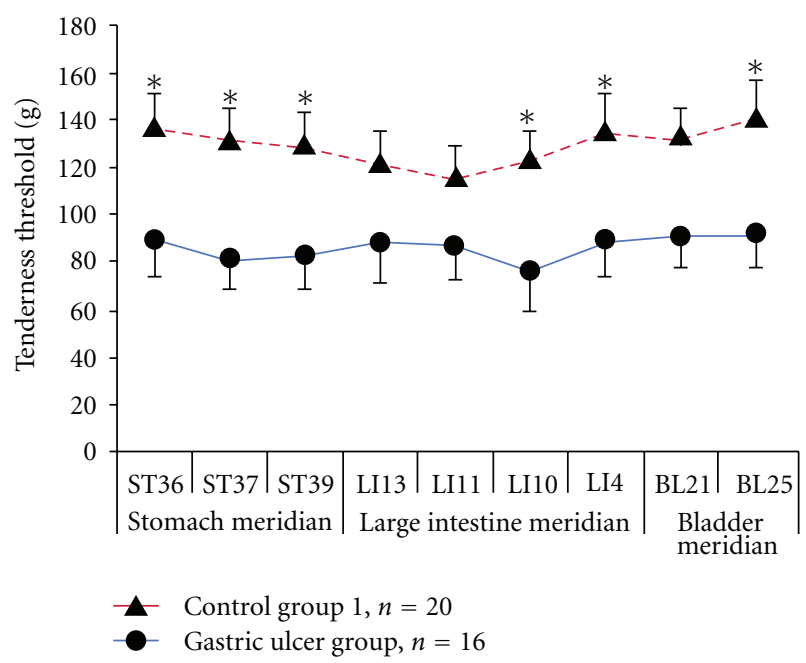

Figure 2: The PPTs of the gastric ulcer group decreased significantly compared with that of the control group.

The PPTs were measured, respectively, in each testing zone on the abdomen and back.

2.5.5. Gastritis Group. Twenty one volunteers with gastritis were selected ( 9 male and 12 female, aged 30-60). The PPTs were measured, respectively, in each testing zone on the abdomen and back.

\section{Results}

\subsection{The Comparison between Gastric Ulcer Group 1 and Con- trol Group 1}

3.1.1. The Comparison of PPTs between the Gastric Ulcer Group and the Control Group. The results showed a significant decrease of PPTs of the test points on related meridians of patients with gastric ulcer in disease state compared with the control group $(P<0.05)$ (Figure 2$)$.

3.1.2. The Comparison of PPTs between the Selected Acupoints and Adjacent Points. There was no significant difference in PPTs between the points along the tested meridians and the adjacent open points (Figure 3 ).

\subsection{The Comparison of PPTs among Gastric Ulcer Group 2, Gastritis Group, and Control Group 2}

3.2.1. The Distribution of Tender Points on the Abdomen and Back. The distribution of tender points in the abdominal and back testing zones was detected by using the fingerpressure method. The results showed that there was no apparent tender point in the normal control group, but, in the gastric ulcer group, tender points appeared more often on the left side of the abdomen and the lower right side of the back (Figure 4 ).

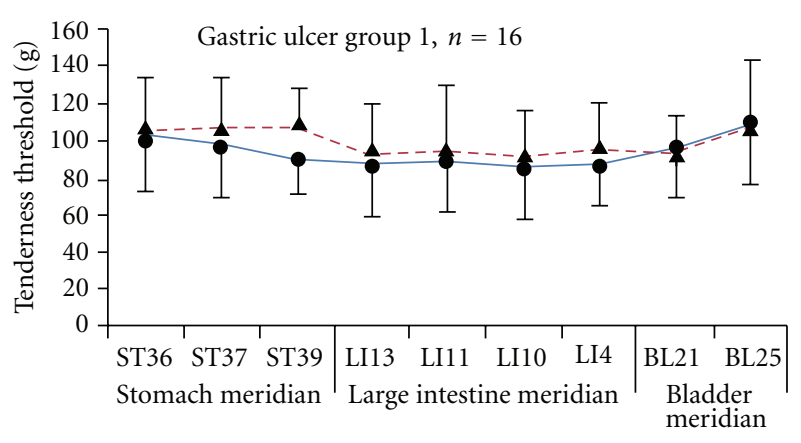

(a)

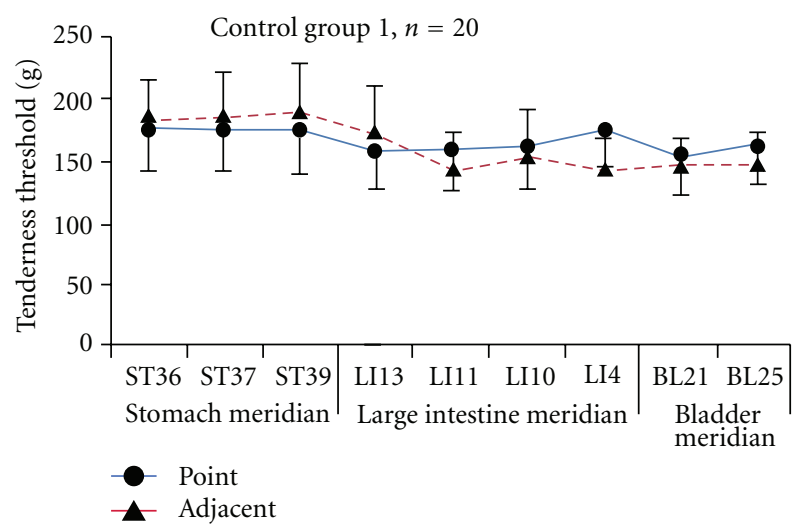

(b)

FIgure 3: There was no significant difference in PPTs between points on meridians and nonmeridians.

3.2.2. The Distribution of the Pain-Sensitive Points on the Abdomen and Back. The PPTs of pain-sensitive points displayed in Figures 5(a) and 5(b) were measured by von Frey detector. Figure 5 shows a dispersed distribution of pain-sensitive points on the abdomen and back in the control group, and there was no relative specificity. The painsensitive points of the gastric ulcer group were relatively concentrated. They were mainly distributed at BURONG (ST19); LIANGMEN (ST21), and HUAROUMEN (ST24), among others of the stomach meridian on the abdomen and PISHU (BL20), WEISHU (BL21), YANGGANG (BL48), WEICANG (BL50), among others of the bladder meridian on the back (Figure 6). The distribution of the pain-sensitive points was basically consistent with the distribution of tender points obtained by using the finger-pressure method. In the gastritis group, the distribution of the pain-sensitive points was less concentrated than that of the gastric ulcer group. It may be attributed to the fact that the clinical symptoms of patients with gastritis were less severe than that of patients with gastric ulcer.

\section{Discussion}

Mechanical tenderness is not only a symptom of local skin or muscle tissue, but also a typical performance of some pain syndromes. It has diagnostic significance in clinical settings. The PPT can be used as an indicator for the evaluation of 


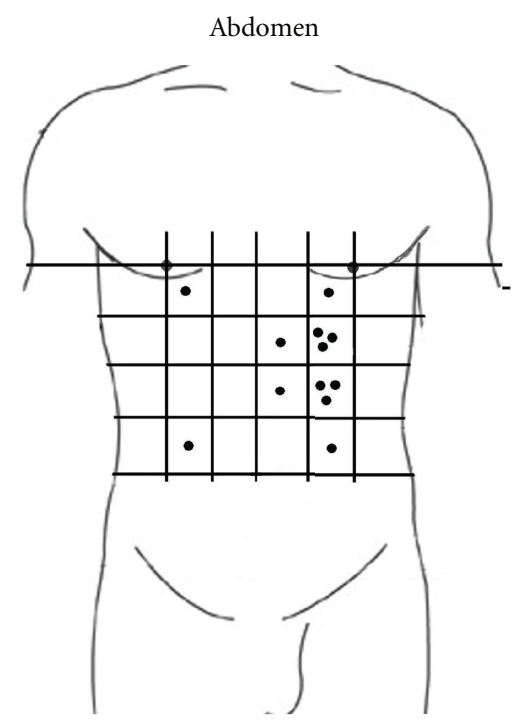

(a)

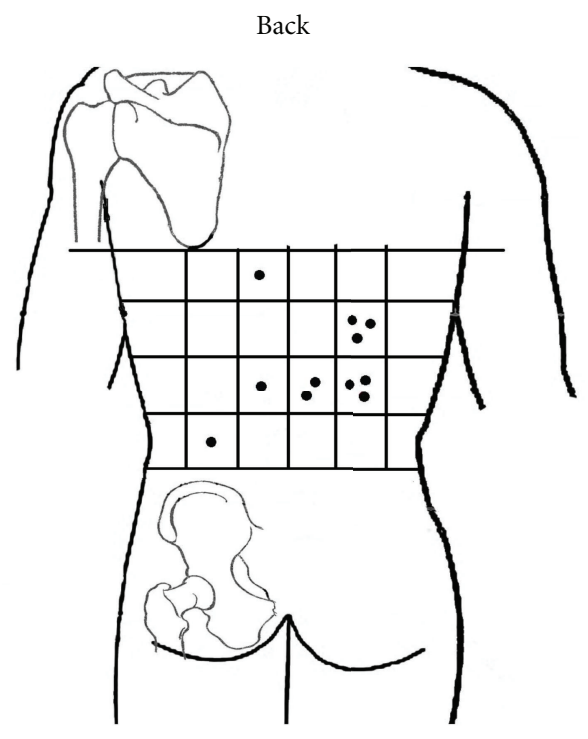

(b)

FIGURE 4: There were more tender points distributed on the left side of the abdomen and the lower right side of the back in the gastric ulcer group.

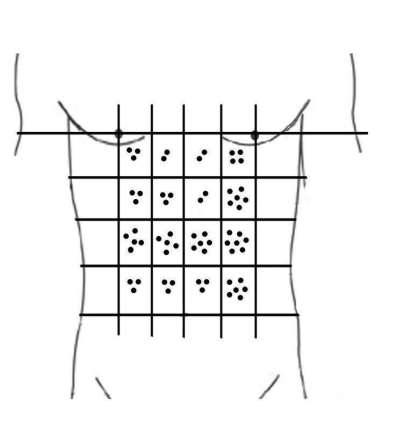

Control group 2

$n=21$

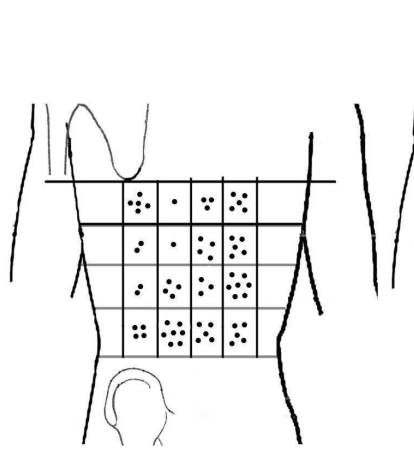

Control group 2

$n=21$

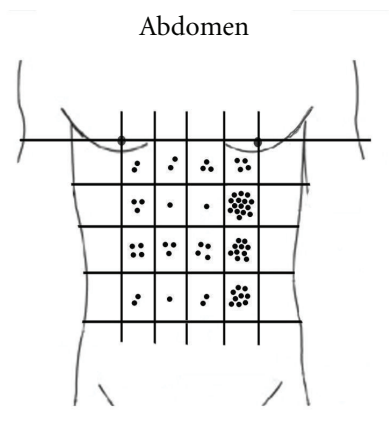

Gastric ulcer group 2

$n=22$

(a)

Back

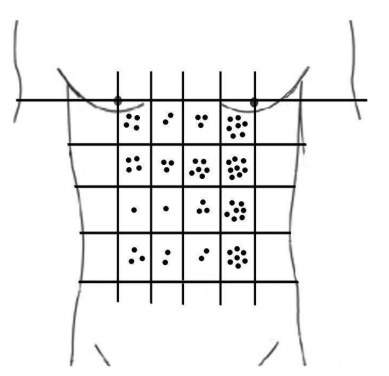

Gastritis group
$n=21$

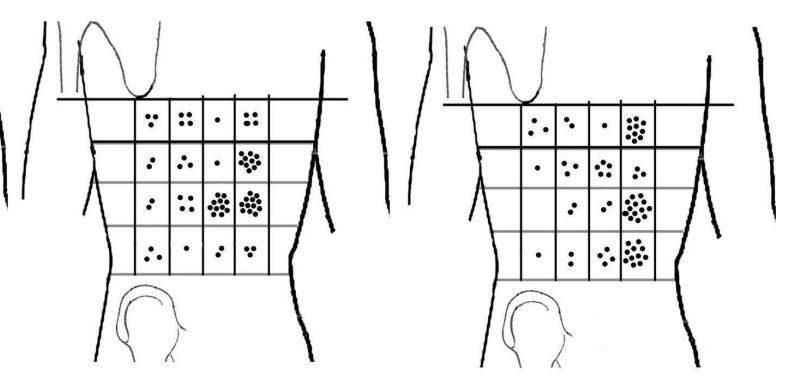

Gastric ulcer group 2

$n=22$

Gastritis group

$n=21$

(b)

FIGURE 5: (a) The distribution of the pain-sensitive points on the abdomen. (b) The distribution of the pain-sensitive points on the back. 


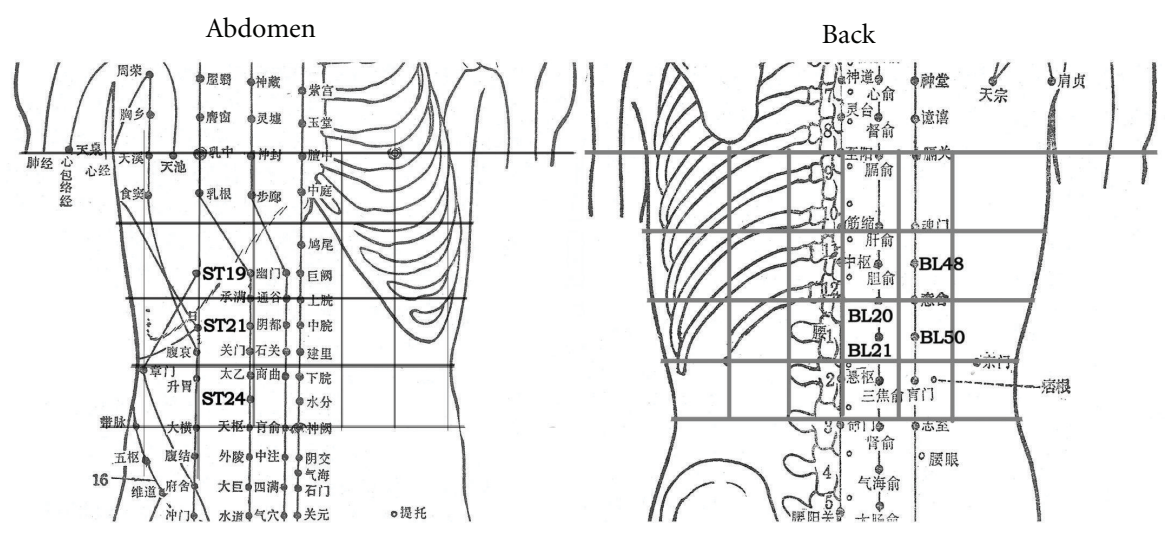

FIGURE 6: The pain-sensitive points were mainly distributed at ST19, ST21, ST24, among others of the stomach meridian on the abdomen and at BL20, BL21, BL48, BL50, among others of the bladder meridian on the back.

the body's inflammatory activity, subcutaneous tissue sensitivity, and pain tolerance. It has been applied in the assessment of the efficacy of various clinical treatment therapies $[1,2]$.

Research has shown that the primary afferent transmission of pain may be affected by the neuroendocrine interaction [3]. As visceral diseases increase the chemical substances that cause pain (5-HT, histamine, peptides, and others) in the body, the pain sensitivity of the patients increases as well [4]. In 1999, British scholar Kosek [5] reported that the PPTs at the muscle-nerve points were significantly lower than the PPTs of "pure" muscle and/or bone. These "muscle-nerve" points are similar to what we call the meridian points.

Meridian points are the reaction points and the treatment points of visceral diseases in traditional Chinese Medicine. The correlation between meridians and internal organs means that the meridians not only have close correlation with their corresponding organs in terms of physiological function, but also demonstrate specific reactions of visceral disorders on the surface of body in pathological states. The therapeutic stimulation on meridians has a regulatory effect on the function of internal organs [6]. In recent years, Yu et al. have proposed the idea that acupoints are "dynamic" and the functioning of acupoints is a dynamic process [7]. He believes that area size of acupoints on the body surface and the function of acupoints are not fixed. On the contrary, the function and area size of acupoints change with the state of the body and the function of their corresponding internal organs. He suggests that acupoints have two states, namely, on/off. The switch between the two states is a dynamic process and reflects the transition of the body from a "dormant" healthy state to an "activated" pathological state. At the same time, the process is accompanied with changes of the microphysical and chemical environments; that is, at the time of visceral disorder, the acupoints turn from the dormant state to the sensitized state. Thus it is possible to regulate the functioning of internal organs by stimulating acupoints.
In recent years, many studies on acupoint specificity have shown that the function and characteristics of acupoints are closely related to the distribution of specific nerves $[8,9]$ and blood vessels $[10,11]$. Acupoints also exhibit unique physical properties, such as optical specificity [12], thermal specificity [13], and electromagnetic specificity [14]. In addition, gene expression in the brain caused by stimulating acupoints has significantly increased comparing with the gene expression caused by stimulating nonacupoints. The result suggests that the effect of acupuncture on acupoints is different from the effect of acupuncture on nonacupoints [15]. Another study has shown that acupuncture can regulate the nitric oxide (NO) content and the activity of nitric oxide synthase (NOS) in body tissues [16-18]. The increase of NO content in the pathological state reflects the activity of the acupoints from one side.

Results showed that the PPTs of skin in the gastric ulcer group were significantly lower than that of the normal control group, regardless of whether it was tested on related meridians or on the adjacent open points. There was no significant difference in PPTs between points on the meridians and adjacent open points. It showed that in pathological states the meridian points were more sensitive and had lower PPTs than in the normal state. Meridian points had no significant difference compared with the adjacent open points. It suggests that acupoint was not just an isolated point but represents a relatively sensitive point area with certain special function. Previous studies have shown that meridian has an optimized and inclusive systematic structure instead of a single channel structure [19].

The distribution of pain-sensitive points on the abdomen and back also suggests that, in the gastric ulcer group, there were obvious tender points in the reflex zones on the body surface associated with the disease. The PPTs were lower, and the pain-sensitive points were more concentrated. Tender points and pain-sensitive points were mainly concentrated at ST19, ST21, and ST24 on the Stomach Meridian of FootYangming of the abdomen and at BL20, BL21, BL48, and BL50 on the Bladder Meridian of Foot-Taiyang of the back. 
A previous research showed that the LIANGMEN point area on the abdomen and the WEISHU point area on the back had a clear overlap with the segmental distribution of nerves of the stomach [20]. The Foot-Yangming Meridian points and the back points were closely related to the stomach $[21,22]$. According to the responses of subjects, they felt apparent pain or tingling when these areas were pressed or tested, and sometimes the string pain goes up and down along the meridian. It is evident that there is a relatively specific link between the meridians (acupoints) on the body surface and the stomach diseases. But based on the feeling of subjects and the tested results, the correlation was not apparent in all states. We observed that there was no tender point on the body of subjects in the normal control group, and the distribution of pain-sensitive points was relatively dispersed. But in pathological states the subjects felt clear pain at meridian points (acupoints). This showed that, in pathological states, acupoints and meridian areas on the body surface were more sensitive and active compared with the normal state. This performance was positively correlated to the alleviation of clinical symptoms and pain $[1,2]$.

The above results suggest that the practical significance of acupoint may lie in its role as a relatively sensitive functional area. The reflex area of internal organs on the surface of body is more sensitive and functionally more active than other areas of the body surface, particularly in pathological states. Therefore, acupoints (point areas) cannot only "mirror the disease" in diagnosis, but also help to "cure the disease" in treatment.

\section{Conclusions}

In conclusion, tender points appeared on the abdomen and back regions of patients with gastric ulcer or gastritis, suggesting that the essence of acupoints goes beyond a mere site for stimulation. Thus, our study provides scientific evidence for the theory of "correlation between meridians and viscera" and, further, helps elucidate the mechanism of acupuncture in the management of gastrointestinal diseases.

\section{Appendix}

The positions of mentioned acupoints in this paper

ST19: on the upper abdomen, 6 cun above the centre of the umbilicus and 2 cun lateral to the anterior midline.

ST21: on the upper abdomen, 4 cun above the centre of the umbilicus and 2 cun lateral to the anterior midline.

ST24: on the upper abdomen, 1 cun above the centre of the umbilicus and 2 cun lateral to the anterior midline.

ST36: on the anteriolateral side of the leg, 3 cun below Dubi (ST35), one finger breadth (middle finger) from the anterior crest of the tibia.
ST37: on the anteriolateral side of the leg, 6 cun below Dubi (ST35), one finger breadth (middle finger) from the anterior crest of the tibia.

ST39: on the anteriolateral side of the leg, 9 cun below Dubi (ST35), one finger breadth (middle finger) from the anterior crest of the tibia.

LI10: 2 cun above Quchi (LI11) when a fist is made.

LI11: between the humerus and radius when the elbow is flexed and the hand is put on the chest.

LI13: 3 cun above the elbow, mid the Zhouliao (LI12), and on the big vessel (probably the cephalic vein), when the elbow is flexed.

LI14: 7 cun above the elbow, at the lower end of the deltoid muscle.

BL20: on the back below the spinous process of the 11 th thoracic vertebra, $1.5 \mathrm{cun}$ lateral to the posterior midline.

BL21: on the back below the spinous process of the 12th thoracic vertebra, $1.5 \mathrm{cun}$ lateral to the posterior midline.

BL25: on the low back, below the spinous process of the 4th lumbar vertebra, 1.5 cun lateral to the posterior midline.

BL48: on the back, below the spinous process of the 10 th thoracic vertebra, $3 \mathrm{cun}$ lateral to the posterior midline.

BL50: on the back, below the spinous process of the 12 th thoracic vertebra, 3 cun lateral to the posterior midline.

\section{Acknowledgments}

This scientific work was supported by a National Basic Research Program of China (973 program, no. 2012CB518503) and a National Natural Science Foundation of China (no. 30772830) Grant to P.-J. Rong. The first two authors (H. Ben and L. Li) contributed equally to this study. P.-J. Rong designed the study and was responsible for obtaining approval by the Institutional Animal Care and Use Committee of the China Academy of Chinese Medical Sciences. Z.-G. Jin, J.-L. Zhang, Y.-H. Li, and X. Li performed the measurements in Beijing. H. Ben and L. Li analyzed the data and wrote the paper. W.-C. Li helped us to modify the manuscript. All authors approved the paper and there is no competing financial interests for this paper.

\section{References}

[1] Y. Huang, G. L. Liu, and S. M. Zhao, "Changes in the stomach and duodenal ulcers in patients with ear tenderness threshold," Chinese Acupuncture \& Moxibustion, vol. 8, pp. 39-40, 1996.

[2] N. H. Wang, "One of the experimental pain evaluation methods: the tenderness threshold. Foreign Medical Sciences," Physical Medicine and Rehabilitation, vol. 24, no. 3, pp. 97-99, 2004. 
[3] Z. F. Zhao, J. H. Lu, Q. Y. Liu et al., "Influence of interaction between endocrine and nervous factors on the pain threshold, calcitonin gene-related peptide immunoreactivity of dorsal root ganglia and spinal dorsal horn in rat," Acta Universitatis Medictnae Tangii, vol. 29, no. 6, pp. 493-496, 2000.

[4] L. Huang, "Effect of cognitive operation on pain threshold and pain tolerance," Chinese Mental Health Journal, vol. 17, no. 4, pp. 261-262, 2003.

[5] E. Kosek, J. Ekholm, and P. Hansson, "Pressure pain thresholds in different tissues in one body region. The influence of skin sensitivity in pressure algometry," Scandinavian Journal of Rehabilitation Medicine, vol. 31, no. 2, pp. 89-93, 1999.

[6] Y. Wang, K. Ji, and X. H. Zang, "Meridian-organs doctrine of progress and discussion of problems," Journal of Clinical Acupuncture and Moxibustion, vol. 21, no. 6, pp. 54-56, 2005.

[7] X. C. Yu, B. Zhu, J. H. Gao et al., "The scientific basis of the points dynamic process," Journal of Traditional Chinese Medicine, vol. 48, no. 11, pp. 17-23, 2007.

[8] k. Liu, W. Wang, A. H. Li et al., "Morphological research of Liver Meridian and effects of capsaicin on genitofemoral nerve discharge frequency in rats," Anatomía Clínica, vol. 11, no. 4, pp. 232-235, 2006.

[9] Y. L. Zhao, X. R. Chang, J. Yan et al., "Study on the specificity of effects of electroacupuncture at acupoints of Stomach Meridian on gastric myoelectric activity and its efferent pathway in the rat," Chinese Archives of Traditional Chinese Medicine, vol. 23, no. 10, pp. 1788-1790, 2005.

[10] F. Liu and E. Y. Chen, "Distribution of vessels in the frontal part of the interosseous membrane of leg and its relationship with points," Shanghai Journal of Traditional Chinese Medicine, vol. 34, no. 12, pp. 40-41, 2000.

[11] X. Mu, H. Q. Duan, W. Chen et al., "Physiological study on the relationship between the essence of acupuncture point and the microvasalium," Chinese Journal of Basic Medicine in Traditional Chinese Medicine, vol. 7, no. 12, pp. 47-52, 2001.

[12] Y. W. Ding, G. H. Ding, X. Y. Shen et al., "Observation on the characters of infrared radiation spectrum of acupoints in normal humans and CHD patients," Journal of Biomedical Engineering, vol. 23, no. 2, pp. 309-312, 2006.

[13] H. P. Deng, X. Y. Shen, and G. H. Ding, "Characteristics of infrared radiation of moxi and meridian-acupoints," Chinese Acupuncture \& Moxibustion, vol. 24, no. 2, pp. 105-107, 2004.

[14] D. Z. Li, S. T. Fu, and X. Z. Li, "Study on the theory and clinical application of meridians (III)," Chinese Acupuncture \& Moxibustion, vol. 25, no. 1, pp. 53-59, 2005.

[15] J. C. Yu, T. Yu, and J. X. Han, "Analysis on difference of acupuncture effects between acupoint and non-acupoint from difference of gene expression," Chinese Acupuncture \& Moxibustion, vol. 22, no. 11, pp. 749-751, 2002.

[16] Z. H. Zhu, Z. Ding, X. M. Tang, and K. X. Ding, "Experimental study on effects of acupuncture at Zusanli (ST36) on NO and NOS in brain tissue of mice of spleen deficiency," Chinese Acupuncture \& Moxibustion, vol. 5, no. 6, pp. 309-312, 2000.

[17] Z. C. Liu, F. M. Sun, M. H. Zhu, Z. Sun et al., "Effects of acupuncture on contents of nitric oxide and nitric oxide synthase in the hippocampus in the obese rat," Chinese Acupuncture \& Moxibustion, vol. 23, no. 1, pp. 52-54, 2007.

[18] W. Y. Liu and B. Bai, "The effect of nitric oxide in brain on pain threshold of rats," Chinese Journal of Behavioral Medical Science, vol. 8, no. 4, pp. 255-257, 2007.

[19] H. Shi, Y. Wan, and J. S. Han, "Sex differences of analgesia induced by $100 \mathrm{~Hz}$ electroacupuncture and intrathecal injection of dynorphin a in rats," Chinese Journal of Pain Medicine, vol. 5, no. 2, pp. 97-101, 1999.
[20] J. Yan and B. Zhu, Basic and Clinic of Acupuncture, Hunan Science and Technology Press, Hunan, China, 2010.

[21] J. S. Li, J. Yan, and X. R. Chang, "Influence of electric acupuncture at acupoints of the foot-yangming meridian on gastric motion and motilin in rabbits," China Journal of Basic Medicine in Traditional Chinese Medicine, vol. 10, no. 3, pp. 36-39, 2004.

[22] J. Yan, X. R. Chang, J. H. Liu et al., "Study on protective action of electroacupuncture at acupoints of the foot-yangming channel on lesion of gastric mucosa in rabbits," Chinese Acupuncture \& Moxibustion, vol. 21, no. 6, pp. 30-32, 2001. 


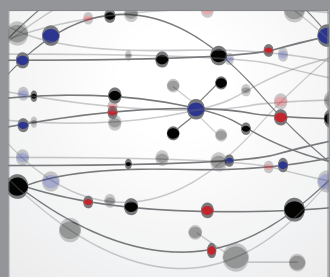

The Scientific World Journal
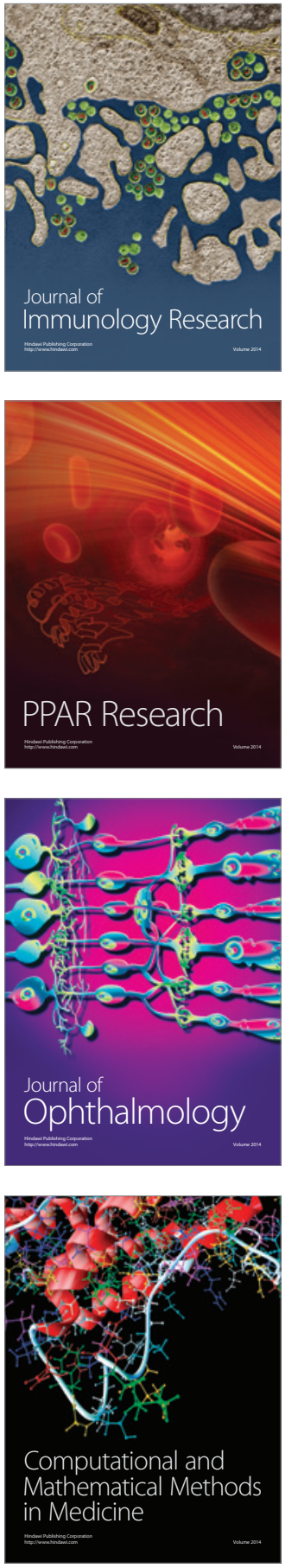

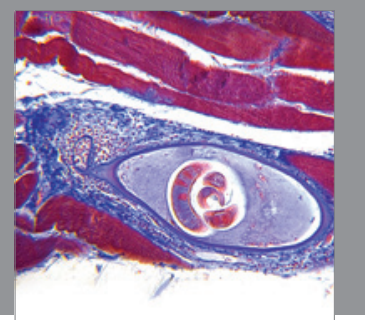

Gastroenterology

Research and Practice
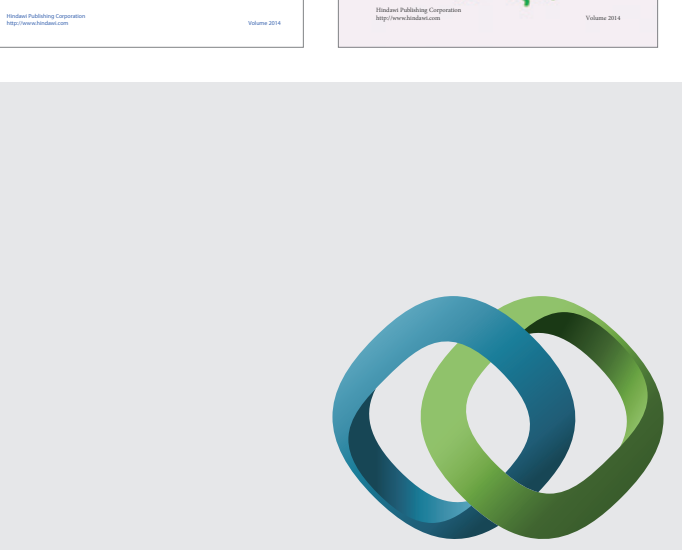

\section{Hindawi}

Submit your manuscripts at

http://www.hindawi.com
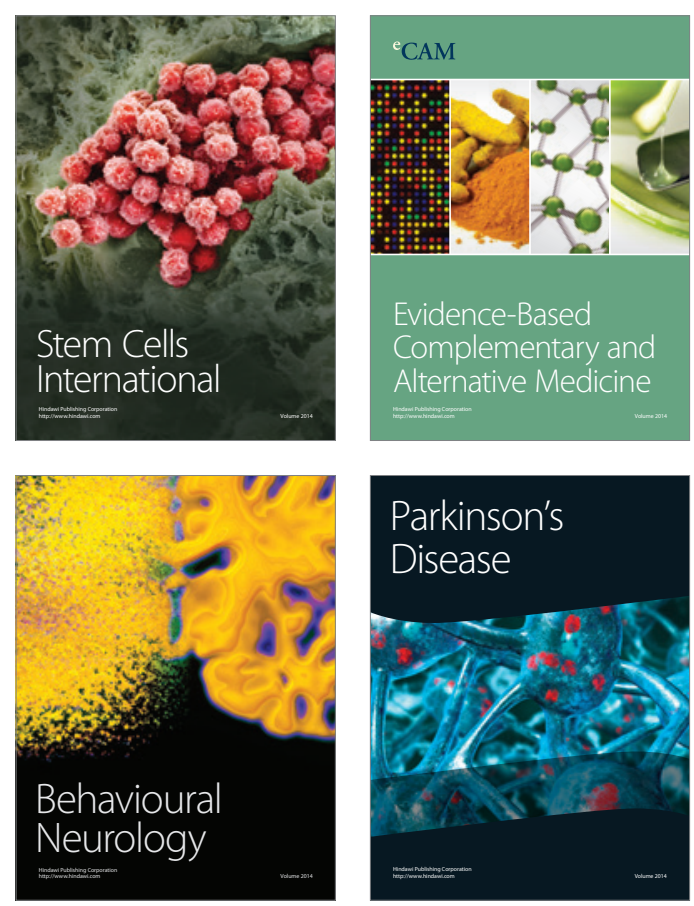

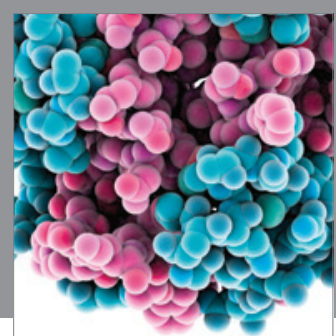

Journal of
Diabetes Research

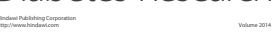

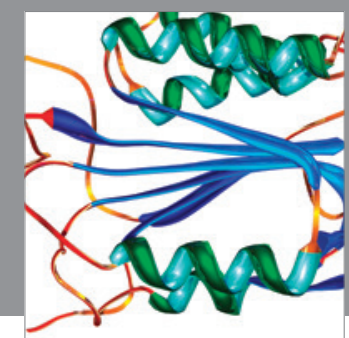

Disease Markers
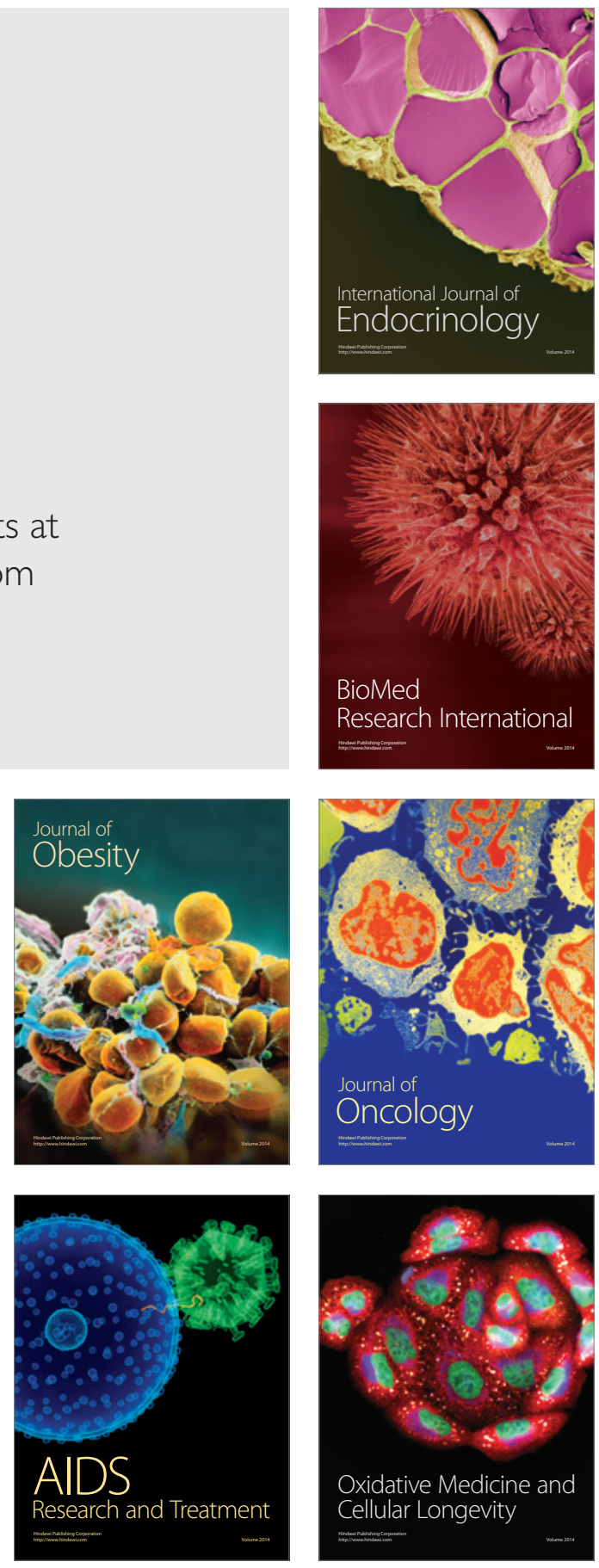